\title{
The Incompressible Navier-Stokes for the Nonlinear Discrete Velocity Models
}

\author{
A BELLOUQUID \\ Department of Mathematics, Politecnico of Torino, \\ Corso Duca degli Abruzzi 24, 10129, Torino, Italy \\ E-mail: bellouq@calvino.polito.it \\ Received September 30, 2001; Revised March 18, 2002; Accepted April 25, 2002
}

\begin{abstract}
We establish the incompressible Navier-Stokes limit for the discrete velocity model of the Boltzmann equation in any dimension of the physical space, for densities which remain in a suitable small neighborhood of the global Maxwellian. Appropriately scaled families solutions of discrete Boltzmann equation are shown to have fluctuations that locally in time converge strongly to a limit governed by a solution of Incompressible Navier-Stokes provided that the initial fluctuation is smooth, and converges to appropriate initial data. As applications of our results, we study the Carleman model and the one-dimensional Broadwell model.
\end{abstract}

\section{Introduction and Main results}

\subsection{Introduction}

The endeavor to understand how fluid dynamical equations can be derived from kinetic theory goes back to the founding works of Maxwell [38] and Boltzmann [16]. Most of derivations are well understood at several formal levels by now, and yet their full mathematical justifications are still missing. Here we establish a so-called incompressible Navier-Stokes fluid dynamical limit for the discrete Boltzmann equation which is an evolution model for a gas which can attain only a finite number of velocities, one has to mention that Inoue and Nishida [30], and Caflish and Papanicolaou [19] have developed an asymptotic theory for the six velocity Broadwell's model. Further Studies have been developed for the two velocity Carleman's model by Kurtz [33] and by Lions and Toscani [36]. The interested reader can recover the pertinent literature in the survey by Lachowicz [34].

As known, see Bonilla and Soler [14], different asymptotic expansions can be developed for kinetic models thus obtaining various hydrodynamic descriptions. It has been recently recognized by several mathematicians, Bardos, Golse and Levermore [3, 4], Demasi, Esposito and Lebowitz [21], that the incompressible Navier-Stokes equation can be obtained as the limit of the Boltzmann equation, when the Knudsen number $\varepsilon>0$ go to zero. The validity of this fluid-dynamical approximation have been studied by several authors, see [2, $5,6,10,21,25,26,37]$. On the other hand, there is non proof concerning the convergence 
of discrete velocity kinetic models to a solution of the incompressible Navier-Stokes equations. However, although a complete proof is not given, some partial results may be found in $[9,10,11,28]$ in particular for Carleman and Broadwell's models. In the present paper a rigorous proof of the connection between these two points of view is done, precising and completing some results which were announced in [4].

Let $\varepsilon>0$ be the Knudsen number, and consider the scaled discrete Boltzmann equation

$$
\varepsilon \partial_{t} F_{i}+v_{i} \cdot \nabla_{x} F_{i}=\frac{1}{\varepsilon} Q_{i}(F, F), \quad i=1, \ldots, m,
$$

where $F_{i}=F_{i}(t, x)$ represents the mass density of gas particles with the constant velocity vector $v_{i}=\left(v_{i}^{1}, \ldots, v_{i}^{n}\right) \in \mathbb{R}^{n}$ at time $t \geq 0$ and position $x=\left(x_{1}, \ldots, x_{n}\right) \in \mathbb{R}^{n} ; Q_{i}$ is a quadratic operator related to the binary collisions:

$$
Q_{i}(F, G)=\frac{1}{2 \alpha_{i}} \sum_{j k l}\left\{A_{k l}^{i j}\left(F_{k} G_{l}+F_{l} G_{k}\right)-A_{i j}^{k l}\left(F_{i} G_{j}+F_{j} G_{i}\right)\right\},
$$

where $\alpha_{i}$ are positive constants, and the terms $A_{i j}^{k l}$ are the so-called transition rates referring to the collisions

$$
\left(v_{i}, v_{j}\right) \leftrightarrow\left(v_{k}, v_{l}\right)
$$

The transition rates are positive constants which, according to the indistinguishability property of the gas particles and the reversibility of the collisions, fulfill the following properties:

$$
A_{l k}^{i j}=A_{k l}^{i j}=A_{k l}^{j i}=\cdots=A_{i j}^{k l} .
$$

A detailed computation of the terms $A_{i j}^{k l}$ can be performed by specializing the velocity discretization and analyzing the related collision mechanics.

The above mathematical structure includes, as particular applications, various mathematical models of discrete Boltzmann equation which can be recovered in the pertinent literature $[18,23]$. It also refers to models with arbitrarily large number of velocities which have been studied in a number of recent papers, see among others [1,27], considering their relatively simpler structure, with respect to the full Boltzmann equation, which may be exploited for scientific computing. Indeed, convergence to the full Boltzmann equation can be studied for models with arbitrary large number of velocities.

Following [7, 18, 23], we shall introduce the basic concepts concerning (1.1) and summarize their properties which will be used later.

Definition 1. A vector $\phi=t_{\left(\phi_{1}, \ldots, \phi_{m}\right)} \in \mathbb{R}^{m}$ is called a summational invariant if

$$
A_{k l}^{i j}\left(\frac{\phi_{i}}{\alpha_{i}}+\frac{\phi_{j}}{\alpha_{j}}-\frac{\phi_{k}}{\alpha_{k}}-\frac{\phi_{l}}{\alpha_{l}}\right)=0
$$

for all $i, j, k, l=1, \ldots, m$.

We denote by $\mathbb{M}$ the set of summational invariants. Then $0<\operatorname{dim} \mathbb{M}<m$ because $t_{\left(\alpha_{1}, \ldots, \alpha_{m}\right)} \in \mathbb{M}$ and $\mathbb{M} \neq \mathbb{R}^{m}$. The following three conditions are equivalent: 
(i) $\phi \in \mathbb{M}$,

(ii) $\langle\phi, Q(F, G)\rangle=0$ for all $F, G \in \mathbb{R}^{m}$,

(iii) $\langle\phi, Q(F, F)\rangle=0$ for all $F \in \mathbb{R}^{m}$.

Here $\langle\cdot, \cdot\rangle$ denotes the standard inner product in $\mathbb{R}^{m}$. For the proof see $[7,18,23]$.

Let $F=t_{\left(F_{1}, \ldots, F_{m}\right)} \in \mathbb{R}^{m}$. We write $F>0$ if $F_{i}>0$ for all $i=1, \ldots, m$.

Definition 2. A vector $F=t_{\left(F_{1}, \ldots, F_{m}\right)}>0$ is called a local Maxwellian if

$$
A_{k l}^{i j}\left(F_{i} F_{j}-F_{k} F_{l}\right)=0, \quad \text { for all } i, j, k, l=1, \ldots, m .
$$

In particular, $F>0$ is called an absolute Maxwellian if it is a locally Maxwellian state and is independent of $t$ and $x$.

Consider the initial value problem for Eq. (1.1):

$$
\begin{aligned}
& \varepsilon \partial_{t} F_{\varepsilon}+\sum_{j=1}^{n} V^{j} \partial_{x_{j}} F_{\varepsilon}=\frac{1}{\varepsilon} Q\left(F_{\varepsilon}, F_{\varepsilon}\right), \quad t>0, \quad x \in \mathbb{R}^{n}, \\
& F_{\varepsilon}(0, x)=F_{0}(x),
\end{aligned}
$$

where

$$
V^{j}=\operatorname{diag}\left(v_{j}^{1}, \ldots, v_{j}^{m}\right), \quad j=1, \ldots, n,
$$

and

$$
Q(F, G)=t_{\left(Q_{1}(F, G), \ldots, Q_{m}(F, G)\right)} .
$$

According to [4], the discrete velocity Boltzmann equation (1.1) gives the incompressible Navier-Stokes equation in the limit $\varepsilon \rightarrow 0$ if $F_{\varepsilon}$ remains near an absolute Maxwellian $M$ with the distance of order $\varepsilon$.

Let $M>0$ be an absolute Maxwellian state, and let

$$
\Lambda=\operatorname{diag}\left(\frac{M_{1}}{\alpha_{1}}, \ldots, \frac{M_{m}}{\alpha_{m}}\right) .
$$

We shall see the solution in the form

$$
F_{\varepsilon}(t, x)=M+\varepsilon \Lambda^{\frac{1}{2}} f_{\varepsilon} .
$$

Then Problem (1.4) can be transformed into the following one:

$$
\begin{gathered}
\partial_{t} f_{\varepsilon}+\frac{1}{\varepsilon} \sum_{j=1}^{n} V^{j} \partial_{x_{j}} f_{\varepsilon}+\frac{1}{\varepsilon^{2}} L f_{\varepsilon}=\frac{1}{\varepsilon} \Gamma\left(f_{\varepsilon}, f_{\varepsilon}\right), \\
f_{\varepsilon}(t=0, x)=f_{0}(x)=\Lambda^{-\frac{1}{2}}\left(F_{0}(x)-M\right),
\end{gathered}
$$

where the operators $L$ and $\Gamma$ are given by

$$
\begin{aligned}
& L f=-2 \Lambda^{-\frac{1}{2}} Q\left(M, \Lambda^{\frac{1}{2}} f\right), \\
& \Gamma(f, g)=\Lambda^{-\frac{1}{2}} Q\left(\Lambda^{\frac{1}{2}} f, \Lambda^{\frac{1}{2}} g\right),
\end{aligned}
$$

and have (see $[7,18,23])$ the following properties: 
(i) $L$ is real symmetric and positive semi-definite. It is null space is given by $N(L)=$ $\Lambda^{\frac{1}{2}} \mathbb{M}$. We denote by $\left\{e_{i}, i=1, \ldots, d\right\}$ the orthonormal basis for $N(L)$.

(ii) $\Gamma$ is bi-linear and satisfies $\Gamma(f, g) \in N(L)^{\perp}$ for any $f, g \in \mathbb{R}^{m}$, where $N(L)^{\perp}$ denotes the orthogonal complement of $N(L)$ in $\mathbb{R}^{m}$.

Existence of global solutions for discrete kinetic models have been obtained by several authors (see [15, 17] and the review article of Bellomo and Gustafsson [8]). However these results cannot be used here because they are based on the dispersion properties of the linearized equations and concern small (with respect to the collision operator) perturbations of the vacuum. It is easy to prove that any finite velocity model has a unique smooth solution during a finite time that depends on the size of the initial data and on the collision operator (or a global existence see [31, 32]). To the best of our knowledge it has not been proven that the time of existence of this smooth solution is independent of $\varepsilon$.

Now we state our main results.

\subsection{Main results}

Our main result is an existence theorem that holds for all $\varepsilon>0$ and a proof of the validity of the fluid-dynamical approximation. To state our result precisely, one needs some function spaces.

Let $C(\Omega, X)$ and $L^{\infty}(\Omega, X)$ denote the spaces of the continuous and bounded functions on $\Omega \subset \mathbb{R}^{n}$ with values in a Banach space $X$, respectively.

$H^{l}$ denotes the $L^{2}\left(\mathbb{R}^{n}\right)$-Sobolev space of order $l$, with the norm $\|\cdot\|_{l}$.

Theorem 1. Let (1.3) be assumed. Let $n \geq 1$ and $l \geq \frac{n}{2}$. If $f_{0} \in H^{l}\left(\mathbb{R}^{n}\right)$, then there exists a positive constants $T_{0}$ and $k$ (depending only on $\left\|f_{0}\right\|_{l}$ ) such that the initial value problem (1.5) has a unique solution $f_{\varepsilon} \in L^{\infty}\left(\left[0, T_{0}\right], H^{l}\right) \cap C\left(\left[0, T_{0}\right], H^{l-1}\left(\mathbb{R}^{n}\right)\right)$ satisfying

$$
\left\|f_{\varepsilon}(t)\right\|_{l} \leq k
$$

for $t \in\left[0, T_{0}\right]$.

If, in addition the initial data satisfies:

$$
\begin{aligned}
& f_{\varepsilon}(0)=h_{\varepsilon}+\varepsilon^{2} k_{\varepsilon} \quad \text { where } \quad k_{\varepsilon} \in H^{l} \quad \text { and, } \\
& h_{\varepsilon} \in N(L), \quad\left\|\Gamma\left(h_{\varepsilon}, h_{\varepsilon}\right)\right\|_{l-1} \leq C \varepsilon, \quad\left\|\partial_{x} h_{\varepsilon}\right\|_{l-1} \leq C \varepsilon, \\
& \lim _{\varepsilon \rightarrow 0}\left\|h_{\varepsilon}-h\right\|_{l-1}=0 .
\end{aligned}
$$

one gets the strong convergence for the discrete Boltzmann equation to Incompressible Navier-Stokes equations:

Theorem 2. Let $f_{\varepsilon}$ be as in Theorem 1. Then, as $\varepsilon \rightarrow 0, f_{\varepsilon} \rightarrow f$ weakly $\star$ in $L^{\infty}\left([0, T], H^{l}\right)$ and strongly in $C\left([0, T], H^{l-1}\right)$ for any $T>0$, and the limit has the form

$$
f=\sum_{i=1}^{d} \rho_{i} e_{i},
$$


where $\rho_{i}=\left\langle e_{i}, f\right\rangle$ satisfy:

$$
\nabla_{x} \cdot\langle V e \otimes e \cdot \rho\rangle=0 .
$$

Moreover, $\rho(t, x)$ is a weak solution of the equations:

$$
\begin{aligned}
& \frac{\partial \rho}{\partial t}-\nabla^{2}:\left(\left\langle L^{-1}\left(P^{\perp}(V e)\right) \otimes(V e)\right\rangle \cdot \rho\right)+\frac{1}{2} \nabla \cdot\left\langle P^{\perp}(V e) \frac{(\rho \cdot e)^{2}}{\sqrt{\alpha M}}\right\rangle=\langle V \cdot \nabla e \otimes e\rangle \cdot \pi \\
& \rho(t=0)=\langle h, e\rangle
\end{aligned}
$$

where $\frac{g^{2}}{\sqrt{\alpha M}}$ denotes the vector $\left(\frac{g_{1}^{2}}{\sqrt{\alpha_{1} M_{1}}}, \ldots, \frac{g_{n}^{2}}{\sqrt{\alpha_{n} M_{n}}}\right)$ and the vectors

$$
\nabla^{2}:\left\langle V e \otimes L^{-1}\left(P^{\perp}(V e)\right)\right\rangle \rho \quad \text { and } \quad \nabla \cdot\left\langle P^{\perp}(V e), \frac{(\rho \cdot e)^{2}}{\sqrt{\alpha M}}\right\rangle
$$

are given by:

$$
\begin{aligned}
& \nabla^{2}:\left\langle V e \otimes L^{-1}\left(P^{\perp}(V e)\right)\right\rangle \rho=\left(\sum_{j=1}^{n} \sum_{k=1}^{n} \partial_{x_{j}} \partial_{x_{k}}\left\langle V^{k} \rho \cdot e, L^{-1}\left(P^{\perp}\left(V^{j} e_{i}\right)\right)\right\rangle\right)_{i} \\
& \nabla \cdot\left\langle P^{\perp}(V e), \frac{(\rho \cdot e)^{2}}{\sqrt{\alpha M}}\right\rangle=\left(\sum_{j=1}^{n} \partial_{x_{j}}\left\langle\frac{(\rho \cdot e)^{2}}{\sqrt{\alpha M}}, P^{\perp}\left(V^{j} e_{i}\right)\right\rangle\right)_{i}
\end{aligned}
$$

Let $\left(\rho_{\varepsilon}\right)_{i}=\left\langle e_{i}, f_{\varepsilon}\right\rangle$. Since $\left\{e_{i}, i=1, \ldots, d\right\}$ forms an orthogonal system, $\rho$ in (1.10) is given by $\rho_{i}=\left\langle e_{i}, f\right\rangle$. One gets

Theorem 3. Let (1.9) be assumed. Then, as $\varepsilon \rightarrow 0, \rho_{\varepsilon} \rightarrow \rho$ weakly $\star$ in $L^{\infty}\left([0, T], H^{l}\right)$ and strongly in $C\left([0, T], H^{l-1}\right)$ for any $T>0$, and the limit $\rho$ satisfies the incompressible Navier-Stokes equations (1.11)-(1.12).

The system (1.11)-(1.12) are generalizations of the Navier-Stokes equations. The condition (1.11) generalizes the $\nabla \cdot u=0, \nabla(\rho+\theta)=0$ conditions that arise in the classical incompressible Navier-Stokes limit (see [3]). $\pi$ is the lagrange multiplier corresponding to the constraint (1.11) and is the generalization of the classical pressure term. The quadratic term in (1.12) corresponds to the classical convection terms. The justification of these formal approximations for the classical Boltzmann equation has proven difficult because many regularity questions remain open for both these fluid systems as the Boltzmann equation. Two approaches to circumventing these difficulties have emerged recently. First some authors have studied direct derivations of linear or weakly nonlinear fluid dynamical systems, such as incompressible Navier-Stokes [21]. Their result requires smooth initial data and holds for as long as the limiting solution of the incompressible Navier-Stokes system is smooth. Second, some authors have abandoned the traditional expansion-based derivations in favor of moments based formal derivation $[5,6,26]$. In [6] it is shown that the solution of the Boltzmann equation considered over space of dimension three or more will be smooth for all time, with small initial data and will converge strongly to the solution of the incompressible Navier-Stokes equations. Recently the Incompressible Navier-Stokes fluid dynamical limit for the classical Boltzmann equation is considered 
in [26]. It was shown that the scaled families of DiPerna-Lions renormalized solutions [22] have fluctuations that globally in time converge weakly to a limit governed by a solution of the incompressible Navier-Stokes equations due to Leray [35] provided that

$$
H\left(f_{\varepsilon}(0)\right)=\iint_{\mathbb{R}^{3} \times \mathbb{R}^{3}}\left(f_{\varepsilon}(0) \log f_{\varepsilon}(0)-f_{\varepsilon}(0)+1\right) M d v d x \leq C \varepsilon^{2},
$$

and the fluid moments of their initial fluctuations converge to appropriate $L^{2}$ initial data. The proof use the averaging lemma (cf. [24]). The averaging lemma is valid for continuous solutions and has no counterpart for discrete velocity models (except in one space dimension (cf. Tartar [39])). However, in the proof of Theorem 2, one needs to evaluate the limit of the nonlinear moment

$$
\left\langle L^{-1}\left(P^{\perp}(V e)\right), \Gamma\left(f_{\varepsilon}, f_{\varepsilon}\right)\right\rangle .
$$

Some uniform regularity estimates would likely be needed for obtaining the limit of nonlinear terms (1.13). This term disappears in the case of Stokes limit (see [12]).

Here we establish a so-called incompressible Navier-Stokes fluid dynamical limit (1.11)(1.12) for the discrete Boltzmann equation in any dimension of the physical space. We solve (1.5) by using the principle of contraction mappings, we introduce the iteration scheme (Lemma 1 and Lemma 2) similar to one used in [19] to obtain the uniform estimate of the remainder term in the Hilbert expansion in order to justify the compressible Euler system from the one dimensionnal Broadwell. The convergence of the scheme to a solution of equation (1.5) is proved by using some properties of the operators $L$ and $\Gamma$. The strong convergence of the solution of equation (1.5) as $\varepsilon \rightarrow 0$ is proved by the uniform estimate and the equicontinuity in $t \in[0, T]$ of the solution with respect to $\varepsilon \in(0,1)$ (Lemma 3) provided that the initial fluctuation is smmoth, close to an $N(L)$ element which converges to appropriate initial data.

Remark 1. Put $h_{\varepsilon}=\Lambda^{\frac{1}{2}} t_{\left(\alpha_{1}, \alpha_{2}, \ldots, \alpha_{m}\right)} w_{\varepsilon}$, one gets

$$
\Gamma\left(h_{\varepsilon}, h_{\varepsilon}\right)=w_{\varepsilon}^{2} \Lambda^{-\frac{1}{2}} Q(M, M)=0 .
$$

An example of assumptions (1.9) can be given by:

$$
\begin{aligned}
& f_{\varepsilon}=\Lambda^{\frac{1}{2}} t_{\left(\alpha_{1}, \alpha_{2}, \ldots, \alpha_{m}\right)} w_{\varepsilon}+\varepsilon^{2} k, \\
& \left\|\partial_{x} w_{\varepsilon}\right\|_{l-1} \leq C \varepsilon, \quad \lim _{\varepsilon \rightarrow 0}\left\|w_{\varepsilon}-w\right\|_{l-1}=0 .
\end{aligned}
$$

Remark 2. In the case where $\frac{h_{\varepsilon}^{2}}{\sqrt{\alpha M}} \in N(L)$, one has $\Gamma\left(h_{\varepsilon}, h_{\varepsilon}\right)=0$ (see Lemma 5). Therefore an other example can be given by:

$$
\begin{aligned}
& f_{\varepsilon}(0)=h_{\varepsilon}+\varepsilon^{2} k_{\varepsilon}, \quad \text { where } \quad k_{\varepsilon} \in H^{l} \quad \text { and, } \\
& h_{\varepsilon} \in N(L), \quad \frac{h_{\varepsilon}^{2}}{\sqrt{\alpha M}} \in N(L), \quad\left\|\partial_{x} h_{\varepsilon}\right\|_{l-1} \leq C \varepsilon, \quad \lim _{\varepsilon \rightarrow 0}\left\|h_{\varepsilon}-h\right\|_{l-1}=0 .
\end{aligned}
$$

Remark 3. In [12] it was proven that the kinetic models (1.1) converge weakly and strongly to the linearized incompressible Navier-Stokes. The assumption $\Gamma\left(h_{\varepsilon}, h_{\varepsilon}\right)=O(\varepsilon)$ in $H^{l-1}$ is not necessary in this case and the time of existence of solutions goes to infinity as $\varepsilon \rightarrow 0$. 
Remark 4. The present work improves upon the result given in [12] for the Carleman and Broadwell's model, without assuming some restriction upon the scaling of the fluctuation with respect to Knudsen number, namely that the fluctuations should only be required to be of an order equal to the Knudsen number, but at the cost of being restricted to initial fluctuations (assuming $\Gamma\left(h_{\varepsilon}, h_{\varepsilon}\right)=O(\varepsilon)$ in $H^{l-1}$ ) for the Broadwell model.

The plan of this paper is as follows. Section 1 deals with the above introduction and main results. In Section 2, the formulation of the problem and the proof of uniform existence Theorem are given. In Section 3, the estimate for $\partial_{t} f_{\varepsilon}$ is shown. This estimate is used to prove the strong convergence of the solution to the solution of the non-linear incompressible Navier-Stokes equation. Finally, Sections 4 and 5 contains some applications. As applications of our results, we are dealt with the Carleman model and the one-dimensional Broadwell model.

\section{Uniform existence}

To prove the existence of local solutions to (1.5), one has to get suitable "apriori" estimate.

\section{$2.1 \quad$ Estimates}

Lemma 1. Let $z(t, x)$ be a given function of $t$ and $x$ such that,

$$
\|z(t)\|_{l} \leq k
$$

and let $f(t, x)$ satisfy the linear system

$$
\begin{aligned}
& \partial_{t} f+\frac{1}{\varepsilon} \sum_{j=1}^{n} V^{j} \partial_{x_{j}} f+\frac{1}{\varepsilon^{2}} L f=\frac{1}{\varepsilon} \Gamma(z, f), \\
& f(0, x)=f_{0}(x) .
\end{aligned}
$$

Then there exist $T_{0}$ such that a constant $k$ can be chosen such that

$$
\sup _{0 \leq t \leq T_{0}}\|f(t)\|_{l} \leq k
$$

Proof. From the theory of linear hyperbolic systems we know that (2.2) has a unique solution in $L^{\infty}\left([0, T], H^{l}\right)$ with $\frac{d f}{d t}$ in $L^{\infty}\left([0, T], L^{2}\right)$. X-Mozilla-Status: 0000

Taking the Fourier transform of (2.2) in $x$ yields

$$
\partial_{t} \hat{f}+\frac{1}{\varepsilon} \sum_{j=1}^{n} V^{j} i \zeta_{j} \hat{f}+\frac{1}{\varepsilon^{2}} L \hat{f}=\frac{1}{\varepsilon} \hat{\Gamma}(z, f) .
$$

Take the inner product (in $\mathbb{C}^{m}$ ) of (2.4) with $\hat{f}$. Since $\sum_{j=1}^{n} V^{j} \zeta_{j}$ and $L$ are real symmetric, the real part of $(2.4)$ is

$$
\frac{\partial_{t}|\hat{f}|^{2}}{2}+\frac{1}{\varepsilon^{2}}\langle L \hat{f}, \hat{f}\rangle=\frac{1}{\varepsilon} \operatorname{Re}\langle\hat{\Gamma}(z, f), \hat{f}\rangle,
$$


where $\langle\cdot, \cdot\rangle$ denotes the standard inner product in $\mathbb{C}^{m}$.

Noting that $L$ is positive semi-definite and $\Gamma \in N(L)^{\perp}$, one obtains due to (2.5) and to the the inequality $a b \leq \frac{1}{2}\left(a^{2}+b^{2}\right)$, the following estimate:

$$
\frac{\partial_{t}|\hat{f}|^{2}}{2}+\frac{C_{1}}{\varepsilon^{2}}\left|P^{\perp} \hat{f}\right|^{2} \leq \frac{|\hat{\Gamma}(z, f)|\left|P^{\perp} \hat{f}\right|}{\varepsilon} \leq \frac{1}{2 C_{1}}|\hat{\Gamma}(z, f)|^{2}+\frac{C_{1}}{2 \varepsilon^{2}}\left|P^{\perp} \hat{f}\right|^{2},
$$

where $C_{1}$ is a constant and $P^{\perp}$ is the orthogonal projection onto $N(L)^{\perp}$.

In particular (2.6) implies that

$$
\frac{\partial_{t}|\hat{f}|^{2}}{2} \leq \frac{1}{2 C_{1}}|\hat{\Gamma}(z, f)|^{2}
$$

Noting that for $l>\frac{n}{2}$,

$$
\|\Gamma(f, g)\|_{l} \leq C_{2}\|f\|_{l}\|g\|_{l} \quad \forall f, g \in H^{l} .
$$

So by multiplying $(2.7)$ by $\left(1+|\zeta|^{2}\right)^{l}$, integrating over $[0, t] \times \mathbb{R}_{\zeta}^{n}$ and using (2.8). One obtains from Plancherel's Theorem, the following inequality:

$$
\|f\|_{l}^{2} \leq\left\|f_{0}\right\|_{l}^{2}+\frac{C_{2}^{2}}{C_{1}} \int_{0}^{t}\|f(s)\|_{l}^{2}\|z(s)\|_{l}^{2} d s \leq\left\|f_{0}\right\|_{l}^{2}+C k^{2} T \sup _{t \in[0, T]}\|f(t)\|_{l}^{2},
$$

with $C=\frac{C_{2}^{2}}{C_{1}}$.

Let $T_{0}$ and $k$ are such that

$$
T_{0}=\frac{1}{16 C\left\|f_{0}\right\|_{l}^{2}}, \quad k=\frac{1-\sqrt{1-4 \sqrt{C T}\left\|f_{0}\right\|_{l}}}{2 \sqrt{C T}},
$$

for $T \in\left[0, T_{0}\right]$.

Then the desired estimate (2.3) is an immediate consequence of (2.9). Thus the proof of Lemma 1 is completed.

We shall solve (1.5) by using Lemma 1 and the principle of contraction mappings. Define the iteration scheme $\left\{f_{\varepsilon}^{N}\right\}$ by

$$
\begin{aligned}
& f_{\varepsilon}^{0}=f_{0} \\
& \partial_{t} f_{\varepsilon}^{N+1}+\frac{1}{\varepsilon} \sum_{j=1}^{N} V^{j} \partial_{x_{j}} f_{\varepsilon}^{N+1}+\frac{1}{\varepsilon^{2}} L f_{\varepsilon}^{N+1}=\frac{1}{\varepsilon} \Gamma\left(f_{\varepsilon}^{N}, f_{\varepsilon}^{N+1}\right), \\
& f_{\varepsilon}^{N+1}(0, x)=f_{0}(x), \quad N=0,1,2 \ldots
\end{aligned}
$$

Lemma 2. Let $f_{0} \in H^{l}$. Then suitable constants $T_{0}, k$ and $\lambda(\lambda<1)$ exist such that for any $\varepsilon>0$ and for any $t \in\left[0, T_{0}\right]$, the following estimates are satisfied:

$$
\left\|f_{\varepsilon}^{N+1}\right\|_{l} \leq k
$$

and

$$
\left\|f_{\varepsilon}^{N+1}-f_{\varepsilon}^{N}\right\|_{l} \leq C_{0} \lambda^{\frac{n}{2}}
$$


Proof. Since $\left\|f^{0}\right\|_{l}=\left\|f_{0}\right\|_{l} \leq k,(2.11)$ follows thanks to Lemma 1.

Let $h_{\varepsilon}^{N}=f_{\varepsilon}^{N+1}-f_{\varepsilon}^{N}$, therefore $h_{\varepsilon}^{N}$ satisfy the equation:

$$
\partial_{t} h_{\varepsilon}^{N}+\frac{1}{\varepsilon} \sum_{j=1}^{n} V^{j} \partial_{x_{j}} h_{\varepsilon}^{N}+\frac{1}{\varepsilon^{2}} L f_{\varepsilon}^{N}=\frac{1}{\varepsilon}\left(\Gamma\left(h_{\varepsilon}^{N-1}, f_{\varepsilon}^{N+1}\right)+\Gamma\left(f_{\varepsilon}^{N-1}, h_{\varepsilon}^{N}\right)\right) .
$$

Applying the technique used in the proof of Lemma 1 one gets:

$$
\partial_{t}\left|\hat{h}_{\varepsilon}^{N}\right|^{2} \leq \frac{1}{C}\left(\left|\hat{\Gamma}\left(h_{\varepsilon}^{N-1}, \hat{f}_{\varepsilon}^{N+1}\right)\right|^{2}+\left|\hat{\Gamma}\left(f_{\varepsilon}^{N-1}, \hat{h}_{\varepsilon}^{N}\right)\right|^{2}\right) .
$$

Multiplying by $\left(1+|\zeta|^{2}\right)^{l}$, integrating over $[0, T] \times \mathbb{R}_{\zeta}^{n}$ and using (2.8). One can use again Plancherel's Theorem (2.11) to deduce that:

$$
\left\|h_{\varepsilon}^{N}\right\|_{l}^{2} \leq C k^{2} T\left(\left\|h_{\varepsilon}^{N-1}\right\|_{l}^{2}+\left\|h_{\varepsilon}^{N}\right\|_{l}^{2}\right)
$$

with $C=\frac{C_{2}^{2}}{C_{1}}$.

Since $C k^{2} T<1$, it follows

$$
\left\|h_{\varepsilon}^{N}\right\|_{l}^{2} \leq \frac{k^{2} c T}{1-k^{2} c T}\left\|h_{\varepsilon}^{N-1}\right\|_{l}^{2} .
$$

Put

$$
\lambda=\frac{k^{2} c T}{1-k^{2} c T} .
$$

It is clear that $\lambda<1$ and (2.12) follows from (2.13).

\section{$2.2 \quad$ Proof of Theorem 1}

In view of Lemma 2 the estimates (2.11), (2.12) imply that for each $\varepsilon>0,\left\{f_{\varepsilon}^{N}\right\}$ is a Cauchy sequence in $L^{\infty}\left([0, T], H^{l}\right)$. Let denote its limit by $f_{\varepsilon}(t)$ and note that it satisfies the estimate (1.8), i.e., this limit in $L^{\infty}\left([0, T], H^{l}\right)$.

From (2.10) one sees that $\partial_{t} f_{\varepsilon}^{N+1}$ can be expressed in terms of sequences converging in $L^{\infty}\left([0, T], H^{l-1}\right)$ as $N \rightarrow+\infty$. The limit is

$$
H_{\varepsilon}=-\frac{1}{\varepsilon} \sum_{j=1}^{n} V^{j} \partial_{x_{j}} f_{\varepsilon}-\frac{1}{\varepsilon^{2}} L f_{\varepsilon}+\frac{1}{\varepsilon} \Gamma\left(f_{\varepsilon}, f_{\varepsilon}\right) .
$$

Now let $\Psi(t, x)$ be a $C^{\infty}$ function of compact support in $[0, T] \times K$. We have just seen that

$$
\int_{[0, T] \times K}\left\langle\psi(t, x), \partial_{t} f_{\varepsilon}^{N+1}\right\rangle d t d x \rightarrow \int_{[0, T] \times K}\left\langle\psi(t, x), H_{\varepsilon}(t, x)\right\rangle d t d x
$$

as $N \rightarrow+\infty$. However

$$
\begin{gathered}
\int_{[0, T] \times K}\left\langle\psi(t, x), \partial_{t} f_{\varepsilon}^{N+1}\right\rangle d t d x=-\int_{[0, T] \times K}\left\langle\partial_{t} \psi(t, x), f_{\varepsilon}^{N+1}\right\rangle d t d x \\
\rightarrow-\int_{[0, T] \times K}\left\langle\partial_{t} \psi(t, x), f_{\varepsilon}\right\rangle d t d x,
\end{gathered}
$$


as $N \rightarrow+\infty$. Therefore $H_{\varepsilon}(t)$ is identified with the distributions derivative in $t$ of $f_{\varepsilon}$. It follows that $f_{\varepsilon}$ satisfies the equation (1.5) and moreover $\frac{\partial f_{\varepsilon}}{\partial t} \in L^{\infty}\left([0, T], H^{l-1}\right)$; hence $f_{\varepsilon} \in C\left([0, T], H^{l-1}\right)$. X-Mozilla-Status: 0000

\section{Hydrodynamical limit}

This section deals with the strong convergence of the solution of the discrete velocity model (1.1) towards solution of the nonlinear incompressible Navier-Stokes equations (1.11)-(1.12). In order to derive the hydrodynamical limit, some uniform regularity estimates would likely be needed for obtaining the limit of the nonlinear terms.

\subsection{Strong convergence}

To prove Theorem 2, one needs more than the uniform bound (1.8) for $f_{\varepsilon}$. For this purpose we assume that hypothesis (1.9) holds and we show the uniform bound for $\frac{\partial f_{\varepsilon}}{\partial t}$.

The uniform equicontinuity in $t$ is given by the following:

Lemma 3. Assume that hypothesis (1.9) holds and let $l>\frac{n}{2}+1$. Then

$$
\left\|\frac{d f_{\varepsilon}}{d t}\right\|_{l-1} \leq C \exp \left(\frac{C k^{2} T}{2}\right), \quad \forall t \in[0, T], \quad \varepsilon \in(0,1),
$$

where the constant $C$ does not depend on $\varepsilon$.

Proof. By differentiating the equation (1.5) with respect to t and by taking the Fourier transform of the equation obtained, one has

$$
\frac{d}{d t} \partial_{t} \hat{f}_{\varepsilon}+\frac{1}{\varepsilon} \sum_{j=1}^{n} i \zeta_{j} V^{j} \partial_{t} \hat{f}_{\varepsilon}+\frac{1}{\varepsilon^{2}} L \partial_{t} \hat{f}_{\varepsilon}=\frac{1}{\varepsilon} \partial_{t} \hat{\Gamma}\left(f_{\varepsilon}, f_{\varepsilon}\right) .
$$

Taking the inner product (in $\mathbb{C}^{m}$ ) of $(3.2)$ with $\partial_{t} \hat{f}_{\varepsilon}$. The real part of the resulting equality is

$$
\frac{\partial_{t}\left|\partial_{t} \hat{f}_{\varepsilon}\right|^{2}}{2}+\frac{1}{\varepsilon^{2}}\left\langle L \partial_{t} \hat{f}_{\varepsilon}, \partial_{t} \hat{f}\right\rangle=\frac{1}{\varepsilon} \operatorname{Re}\left\langle\hat{\Gamma}\left(\partial_{t} f, f\right), \partial_{t} \hat{f} .\right\rangle
$$

Since $L$ is positive semi-definite and $\Gamma \in N(L)^{\perp}$, we get

$$
\begin{aligned}
\frac{\partial_{t}\left|\partial_{t} \hat{f}_{\varepsilon}\right|^{2}}{2}+\frac{C_{1}}{\varepsilon^{2}}\left|P^{\perp} \partial_{t} \hat{f}_{\varepsilon}\right|^{2} & \leq \frac{\left|\hat{\Gamma}\left(\partial_{t} f_{\varepsilon}, f_{\varepsilon}\right)\right|\left|P^{\perp} \partial_{t} \hat{f}_{\varepsilon}\right|}{\varepsilon} \\
& \leq \frac{1}{2 C_{1}}\left|\hat{\Gamma}\left(\partial_{t} f_{\varepsilon}, f_{\varepsilon}\right)\right|^{2}+\frac{C_{1}}{2 \varepsilon^{2}}\left|P^{\perp} \partial_{t} \hat{f}_{\varepsilon}\right|^{2} .
\end{aligned}
$$

So, multiplying by $\left(1+|\zeta|^{2}\right)^{l-1}$, integrating over $\mathbb{R}_{\zeta}^{n}$ and using (2.8) one gets

$$
\partial_{t}\left\|\partial_{t} f_{\varepsilon}\right\|_{l-1}^{2} \leq \frac{1}{C_{1}}\left\|\Gamma\left(\partial_{t} f_{\varepsilon}, f_{\varepsilon}\right)\right\|_{l-1}^{2} \leq C\left\|\partial_{t} f_{\varepsilon}\right\|_{l-1}^{2}\left\|f_{\varepsilon}\right\|_{l-1}^{2}
$$


Using Gronwall's inequality, one reduces (3.3) to

$$
\left\|\partial_{t} f_{\varepsilon}\right\|_{l-1} \leq \exp \left(\frac{C k^{2} T}{2}\right)\left\|\partial_{t} f_{\varepsilon}(0)\right\|_{l-1}
$$

By using equation (1.5) in order to express $\partial_{t} f_{\varepsilon}(0)$ in terms of the initial data we have

$$
\left\|\partial_{t} f_{\varepsilon}\right\|_{l-1} \leq \exp \left(\frac{C k^{2} T}{2}\right)\left(\frac{1}{\varepsilon}\left\|\sum_{j=1}^{n} V^{j} \partial_{x_{j}} f_{0}\right\|_{l-1}+\frac{1}{\varepsilon^{2}}\left\|L f_{0}\right\|_{l-1}+\frac{1}{\varepsilon}\left\|\Gamma\left(f_{0}, f_{0}\right)\right\|_{l-1}\right) .
$$

Taking into account hypothesis (1.9), one gets

$$
\begin{aligned}
\left\|\partial_{t} f_{\varepsilon}\right\|_{l-1} \leq & C \exp \left(\frac{C k^{2} T}{2}\right)\left(\frac{1}{\varepsilon}\left\|\partial_{x} h_{\varepsilon}\right\|_{l-1}+\varepsilon\left\|\partial_{x} k_{\varepsilon}\right\|_{l-1}+\left\|L k_{\varepsilon}\right\|_{l-1}\right. \\
& \left.+\varepsilon\left\|h_{\varepsilon}\right\|_{l}\left\|k_{\varepsilon}\right\|_{l}+\varepsilon^{3}\left\|k_{\varepsilon}\right\|_{l}^{2}+\frac{1}{\varepsilon}\left\|\Gamma\left(h_{\varepsilon}, h_{\varepsilon}\right)\right\|_{l-1}\right) \leq C \exp \left(\frac{C k^{2} T}{2}\right) .
\end{aligned}
$$

The proof of (3.1) is now complete.

Conclusion. From Lemma 3 we conclude the following: the solution $f_{\varepsilon}$ is bounded in $C\left([0, T], H^{l-1}\right)$, uniformly for $\varepsilon>0$, for $t$ in any compact subset of the interval $[0, T]$, moreover $f_{\varepsilon}$ satisfies the bound (3.1). Therefore by the Ascoli-Arzela lemma we can choose a convergent subsequence $f_{\varepsilon_{j}}$, where $\varepsilon_{j} \rightarrow 0$ such that

$$
f_{\varepsilon_{j}} \rightarrow f \quad \text { in } \quad C\left([0, T], H^{l-1}\right) .
$$

and the limit function satisfies the bound (1.6).

Thanks to convergence results (3.4), the estimate (1.8) allow us to deduce (possibly taking subsequences) the following convergence

$$
\Gamma\left(f_{\varepsilon_{j}}, f_{\varepsilon_{j}}\right) \rightarrow \Gamma(f, f) \quad \text { strongly in } H^{l-1} .
$$

\subsection{Passage to the limit}

This subsection is devoted to the connection between the discrete velocity kinetic equations (1.1) and the incompressible Navier-Stokes equations (1.11)-(1.12), we use the techniques described in [3]. In order to get these equations, one needs the following:

Lemma 4. Let $f, g \in \mathbb{R}^{m}$. One gets

$$
\langle f, L g\rangle=\frac{1}{8} \sum_{i, j, k, l} A_{k l}^{i j}\left(M_{i} M_{j}+M_{k} M_{l}\right)\left(f_{i}^{\star}+f_{j}^{\star}-f_{k}^{\star}-f_{l}^{\star}\right)\left(g_{i}^{\star}+g_{j}^{\star}-g_{k}^{\star}-g_{l}^{\star}\right),
$$

where

$$
f_{i}^{\star}=\frac{f_{i}}{\sqrt{\alpha_{i} M_{i}}}, \quad i=1, \ldots, m .
$$

Proof. See [7] and [23]. 
Lemma 5. Let $f \in N(L)$. One has

$$
\Gamma(f, f)=\frac{1}{2} L\left(\frac{f^{2}}{\sqrt{\alpha M}}\right) .
$$

Proof. Using (1.7), one gets

$$
\begin{aligned}
\Gamma(f, f) & =\Lambda^{-\frac{1}{2}}\left(\frac{1}{\alpha_{i}} \sum_{j, k, l}\left(A_{k l}^{i j} \sqrt{M_{k} M_{l}} f_{k} f_{l}-A_{i j}^{k l} \sqrt{M_{i} M j} f_{i} f_{j}\right)\right) \\
& =\left(\sum_{j, k, l}\left(A_{k l}^{i j} \frac{\sqrt{M_{k} M_{l}}}{\sqrt{\alpha_{i} \alpha_{k} \alpha_{l} M_{i}}} f_{k} f_{l}-A_{i j}^{k l} \frac{\sqrt{M j}}{\alpha_{i} \sqrt{\alpha_{j}}} f_{i} f_{j}\right)\right)_{i} .
\end{aligned}
$$

On the other hand, if $f \in N(L)$, so one gets by (3.6) the following identity:

$$
A_{k l}^{i j}\left(\frac{f_{i}}{\sqrt{\alpha_{i} M_{i}}}+\frac{f_{j}}{\sqrt{\alpha_{j} M_{j}}}-\frac{f_{l}}{\sqrt{\alpha_{l} M_{l}}}-\frac{f_{k}}{\sqrt{\alpha_{k} M_{k}}}\right)=0, \quad \forall i, j, k, l .
$$

Thus, we have

$$
2 A_{k l}^{i j} \frac{f_{i} f_{j}}{\sqrt{\alpha_{i} \alpha_{j} M_{i} M j}}=A_{k l}^{i j}\left(\frac{f_{l}^{2}}{\alpha_{l} M_{l}}+\frac{f_{k}^{2}}{\alpha_{k} M_{k}}-\frac{f_{i}^{2}}{\alpha_{i} M_{i}}-\frac{f_{j}^{2}}{\alpha_{j} M_{j}}+\frac{2 f_{l} f_{k}}{\sqrt{\alpha_{l} \alpha_{k} M_{l} M k}}\right) .
$$

Hence substituting this identity into (3.8) yields:

$$
\begin{aligned}
\Gamma(f, f)= & \left(\sum _ { j , k , l } A _ { k l } ^ { i j } \left(\frac{\sqrt{M_{k} M_{l}}}{\sqrt{\alpha_{i} \alpha_{l} \alpha_{k} M_{i}}} f_{k} f_{l}-\frac{\sqrt{M_{i}} M_{j}}{\sqrt{\alpha_{i} \alpha_{l} \alpha_{k} M_{k} M_{l}}} f_{k} f_{l}-\frac{1}{2} \frac{\sqrt{M_{i}} M_{j}}{\sqrt{\alpha_{i}} \alpha_{l} M_{l}} f_{l}^{2}\right.\right. \\
& \left.\left.-\frac{1}{2} \frac{\sqrt{M_{i}} M_{j}}{\sqrt{\alpha_{i}} \alpha_{k} M_{k}} f_{k}^{2}+\frac{1}{2} \frac{\sqrt{M_{i}} M_{j}}{\alpha_{i} \sqrt{\alpha_{i}} M_{i}} f_{i}^{2}+\frac{1}{2} \frac{\sqrt{M_{i}}}{\alpha_{j} \sqrt{\alpha_{i}}} f_{j}^{2}\right)\right)_{i}
\end{aligned}
$$

Noting that

$$
A_{k l}^{i j}\left(M_{i} M_{j}-M_{k} M_{l}\right)=0, \quad \forall i, j, k, l .
$$

So,

$$
A_{k l}^{i j}\left(\frac{\sqrt{M_{k} M_{l}}}{\sqrt{\alpha_{i} \alpha_{l} \alpha_{k} M_{i}}} f_{k} f_{l}-\frac{\sqrt{M_{i}} M_{j}}{\sqrt{\alpha_{i} \alpha_{l} \alpha_{k} M_{k} M_{l}}} f_{k} f_{l}\right)=0,
$$

which with (3.9) imply

$$
\begin{gathered}
\Gamma(f, f)=-\frac{1}{2}\left(\sum_{j, k, l} A_{k l}^{i j}\left(\frac{\sqrt{M_{i}} M_{j}}{\sqrt{\alpha_{i}} \alpha_{l} M_{l}} f_{l}^{2}+\frac{\sqrt{M_{i}} M_{j}}{\sqrt{\alpha_{i}} \alpha_{k} M_{k}} f_{k}^{2}-\frac{\sqrt{M_{i}} M_{j}}{\alpha_{i} \sqrt{\alpha_{i}} M_{i}} f_{i}^{2}-\frac{\sqrt{M_{i}}}{\alpha_{j} \sqrt{\alpha_{i}}} f_{j}^{2}\right)\right)_{i} \\
=-\frac{1}{2}\left(\sum_{j, k, l} A_{k l}^{i j}\left(\frac{M_{k}}{\sqrt{\alpha_{i} M_{i}}} \frac{f_{l}^{2}}{\alpha_{l}}+\frac{M_{l}}{\sqrt{\alpha_{i} M_{i}}} \frac{f_{k}^{2}}{\alpha_{k}}-\frac{M_{j}}{\sqrt{\alpha_{i} M_{i}}} \frac{f_{i}^{2}}{\alpha_{i}}-\frac{M_{i}}{\sqrt{\alpha_{i} M_{i}}} \frac{f_{j}^{2}}{\alpha_{j}}\right)\right)_{i} .
\end{gathered}
$$

Therefore the desired identity (3.7) follows from (3.10) and (1.6). 
Lemma 6. Let $\chi$ be a test function such that

$$
\sum_{j=1}^{n} \sum_{r=1}^{d} \partial_{x_{j}} \chi_{r}\left\langle e_{r}, V^{j} e_{l}\right\rangle=0, \quad l=1, \ldots, d
$$

Then

$$
\left\langle\sum_{j=1}^{n} \partial_{x_{j}}\left\langle f_{\varepsilon}, P\left(V^{j}\left(e_{l}\right)\right\rangle, \chi\right\rangle=0, \quad l=1, \ldots, d,\right.
$$

where $P$ is the projection onto $N(L)$.

Proof. One has

$$
\begin{aligned}
& \left\langle\sum_{j=1}^{n} \partial_{x_{j}}\left\langle f_{\varepsilon}, P\left(V^{j}\left(e_{l}\right)\right\rangle, \chi\right\rangle=\left\langle\sum_{j=1}^{n} \partial_{x_{j}}\left\langle P f_{\varepsilon}, V^{j}\left(e_{l}\right)\right\rangle, \chi\right\rangle\right. \\
& \quad=\sum_{j=1}^{n}\left\langle\partial_{x_{j}}\left\langle\sum_{r=1}^{d} \alpha_{\varepsilon, r} e_{r}, V^{j}\left(e_{l}\right)\right\rangle, \chi\right\rangle=\sum_{j=1}^{n} \sum_{r=1}^{d} \sum_{l=1}^{d}\left\langle\partial_{x_{j}} \alpha_{\varepsilon, r}\left\langle e_{r}, V^{j}\left(e_{l}\right)\right\rangle, \chi_{l}\right\rangle .
\end{aligned}
$$

Integrating by parts and using (3.5), one gets

$$
\left\langle\sum_{j=1}^{n} \partial_{x_{j}}\left\langle f_{\varepsilon}, P\left(V^{j}\left(e_{l}\right)\right\rangle, \chi\right\rangle=-\sum_{j=1}^{n} \sum_{r=1}^{d} \sum_{l=1}^{d}\left\langle\alpha_{\varepsilon, r}\left\langle e_{r}, V^{j}\left(e_{l}\right)\right\rangle, \partial_{x_{j}} \chi_{l}\right\rangle=0 .\right.
$$

This ends the proof of Lemma 6 .

Completion of the proof. Multiplying the equation (1.5) by $\varepsilon^{2}$, letting $\varepsilon$ go to zero and using (3.4), yields the relation

$$
L f=0 .
$$

This implies that $f \in N(L)$ and thus can be written according to the formula (1.10).

The scaled local conservation laws associated with the kinetic equation (1.5) are

$$
\partial_{t}\left\langle f_{\varepsilon}, e_{i}\right\rangle+\frac{1}{\varepsilon} \sum_{j=1}^{n} \partial_{x_{j}}\left\langle V^{j} f_{\varepsilon}, e_{i}\right\rangle=0, \quad i=1, \ldots, d .
$$

Letting $\varepsilon$ tend to zero and applying formula (1.8) yields condition (1.11).

As was done in [3] for the classical continuous Boltzmann equation, we divide by $\varepsilon$ and we decompose the flux in the form

$$
\partial_{t}\left\langle f_{\varepsilon}, e_{i}\right\rangle+\frac{1}{\varepsilon} \sum_{j=1}^{n} \partial_{x_{j}}\left\langle f_{\varepsilon}, P^{\perp}\left(V^{j} e_{i}\right)\right\rangle+\frac{1}{\varepsilon} \sum_{j=1}^{n} \partial_{x_{j}}\left\langle f_{\varepsilon}, P\left(V^{j} e_{i}\right)\right\rangle=0 .
$$

Using 3.11 (Lemma 6), the second term of (3.14) can be eliminated upon integrating (3.14) against test functions satisfying (3.11). 
The limit of the first flux term in (3.14) is computed using the fact that $P^{\perp}\left(V^{j} e_{i}\right) \in$ $\operatorname{Ran}(L)$ along with the kinetic equation (1.5) to obtain

$$
\begin{aligned}
\partial_{t}\left\langle f_{\varepsilon}, e_{i}\right\rangle & +\sum_{j=1}^{n} \partial_{x_{j}}\left\langle-\varepsilon \partial_{t} f_{\varepsilon}-\sum_{k=1}^{n} V^{k} \partial_{x_{k}} f_{\varepsilon}+\Gamma\left(f_{\varepsilon}, f_{\varepsilon}\right), L^{-1} P^{\perp}\left(V^{j} e_{i}\right)\right\rangle \\
& +\frac{1}{\varepsilon} \sum_{j=1}^{n} \partial_{x_{j}}\left\langle f_{\varepsilon}, P\left(V^{j} e_{i}\right)\right\rangle=0, \quad i=1, \ldots, d .
\end{aligned}
$$

Letting $\varepsilon$ goes to zero,

$$
\begin{aligned}
& \sum_{j=1}^{n} \sum_{k=1}^{n} \partial_{x_{j}} \partial_{x_{k}}\left\langle V^{k} f_{\varepsilon}, L^{-1}\left(P^{\perp}\left(V^{j} e_{i}\right)\right)\right\rangle \rightarrow \sum_{j=1}^{n} \sum_{k=1}^{n} \partial_{x_{j}} \partial_{x_{k}}\left\langle V^{k} \rho \cdot e, L^{-1}\left(P^{\perp}\left(V^{j} e_{i}\right)\right)\right\rangle \\
& \quad=\nabla^{2}:\left\langle V e \otimes L^{-1}\left(P^{\perp}(V e)\right)\right\rangle \rho,
\end{aligned}
$$

in $D_{t, x}^{\prime}$.

The limiting quadratic term of (3.15) must be evaluated further in order to bring it into the form that appears in (1.12). Since $f \in N(L)$ the convergence (3.5) and the identity (3.7) can be employed to show the following convergence in $D_{t, x}^{\prime}$ as $\varepsilon \rightarrow 0$,

$$
\begin{gathered}
\sum_{j=1}^{n} \partial_{x_{j}}\left\langle\Gamma\left(f_{\varepsilon}, f_{\varepsilon}\right), L^{-1} P^{\perp}\left(V^{j} e_{i}\right)\right\rangle \rightarrow \sum_{j=1}^{n} \partial_{x_{j}}\left\langle\Gamma(f, f), L^{-1} P^{\perp}\left(V^{j} e_{i}\right)\right\rangle \\
=\frac{1}{2} \sum_{j=1}^{n} \partial_{x_{j}}\left\langle\frac{(\rho \cdot e)^{2}}{\sqrt{\alpha M}}, P^{\perp}\left(V^{j} e_{i}\right)\right\rangle=\frac{1}{2} \nabla \cdot\left\langle P^{\perp}(V e), \frac{(\rho \cdot e)^{2}}{\sqrt{\alpha M}}\right\rangle .
\end{gathered}
$$

Passing to the limit $\varepsilon \rightarrow 0$ in equation (3.15), the above convergence (3.16) and (3.17) suffices to write the limit equation (1.12).

By integrating the equation (3.15) over $t$, we have

$$
\begin{aligned}
\left\langle f_{\varepsilon}, e_{i}\right\rangle & -\left\langle f_{\varepsilon}(0), e_{i}\right\rangle \\
= & -\int_{0}^{t}\left(\sum_{j=1}^{n} \partial x_{j}\left\langle-\varepsilon \partial_{t} f_{\varepsilon}-\sum_{k=1}^{n} V^{k} \partial_{x_{k}} f_{\varepsilon}+\Gamma\left(f_{\varepsilon}, f_{\varepsilon}\right), L^{-1} P^{\perp}\left(V^{j} e_{i}\right)\right\rangle\right. \\
& \left.+\frac{1}{\varepsilon} \sum_{j=1}^{n} \partial_{x_{j}}\left\langle f_{\varepsilon}, P\left(V^{j} e_{i}\right)\right\rangle(s)\right) d s=0, \quad i=1, \ldots, d .
\end{aligned}
$$

Let $\varepsilon \rightarrow 0$ and putting $t=0$, it follows

$$
\left\langle f, e_{i}\right\rangle(0)=\left\langle h, e_{i}\right\rangle
$$

Therefore the desired initial condition for the system (1.12) follows from (3.18).

In the next, we will apply the results discussed in Section 1 to two examples. 


\section{Example, I (Carlemann model)}

The simplest one-dimensional discrete-velocity models of the Botzmann equation are certainly those with two velocities. These models describe the evolution of the velocity distribution of a fictitious gas composed of two kinds of particles that move parallel to the $x$-axis with constant speed equal to one, either in the positive $x$-direction with a density $F_{1}$, or in the negative $x$-direction with a density $F_{2}$. The corresponding dimensionless hyperbolic system is given by (see [20]):

$$
\begin{aligned}
& \varepsilon \frac{d F_{1}^{\varepsilon}}{d t}+\frac{d F_{1}^{\varepsilon}}{d x}=\frac{1}{\varepsilon}\left(F_{2}^{\varepsilon 2}-F_{1}^{\varepsilon 2}\right), \\
& \varepsilon \frac{d F_{2}^{\varepsilon}}{d t}-\frac{d F_{2}^{\varepsilon}}{d x}=\frac{1}{\varepsilon}\left(F_{1}^{\varepsilon 2}-F_{2}^{\varepsilon 2}\right), \\
& F_{i}^{\varepsilon}(t=0, x)=F_{i}^{0}, \quad i=1.2 .
\end{aligned}
$$

Here we have $\left(\alpha_{1}, \alpha_{2}\right)=(1,1)$ and

$$
A_{11}^{22}=A_{22}^{11}=1, \quad \text { and } \quad A_{k l}^{i j}=0 \quad \text { otherwise. }
$$

The condition (1.3) is satisfied.The space $M$ of summational invariants consists of vectors $\phi=t_{\left(\phi_{1}, \phi_{2}\right)}$ satisfying $\phi_{1}-\phi_{2}=0$. Therefore $M$ is spanned by $\phi=(1,1)$.

On the other hand a locally Maxwellian state is a vector $F=t_{\left(F_{1}, F_{2}\right)}>0$ satisfying $F_{2}^{2}=F_{1}^{2}$. Let $M=(1,1)$ be an absolute Maxwellian state: $M=(1,1)$. Set $F(t, x)=$ $M+\varepsilon \Lambda^{\frac{1}{2}} f(t, x)$, here $\Lambda=I_{d}$, and substitute it into (4.1), we get:

$$
\begin{aligned}
& \partial_{t} f_{\varepsilon}+\frac{1}{\varepsilon} \partial_{x} V f_{\varepsilon}+\frac{1}{\varepsilon^{2}} L f_{\varepsilon}=\frac{1}{\varepsilon} \Gamma\left(f_{\varepsilon}, f_{\varepsilon}\right), \\
& f_{\varepsilon}(t=0, x)=f_{0}(x),
\end{aligned}
$$

where

$$
L=2\left(\begin{array}{cc}
1 & -1 \\
-1 & 1
\end{array}\right), \quad V=\left(\begin{array}{cc}
1 & 0 \\
0 & -1
\end{array}\right)
$$

and

$$
\Gamma(f, f)=\left(f_{1}^{2}-f_{2}^{2}\right) t_{(-1,1)} .
$$

As $\varepsilon \rightarrow 0$, we get that $f_{\varepsilon} \rightarrow f$ in $D_{t, x}^{\prime}$ (distribution sense) with $f=\rho \cdot e$ and $\rho$ is solution of the heat equation:

$$
\partial_{t} \rho=\frac{1}{4} \partial_{x}^{2} \rho
$$

It follows from the uniqueness of the solution of the initial value problem for (4.3) that all sequences of $f_{\varepsilon}$ as $\varepsilon \rightarrow 0$ give the same system (4.3) in the limit.

Thus we have proved

Theorem 4. Let $f_{0} \in H^{1}(\mathbb{R})$, then there exists a positive constants $T_{0}$ and $k$ (depending only on $\left\|f_{0}\right\|_{l}$ ) such that the initial value problem (4-2) has a unique solution $f_{\varepsilon} \in L^{\infty}\left(\left[0, T_{0}\right], H^{1}(\mathbb{R})\right) \cap C\left([0, T], L^{2}(\mathbb{R})\right)$ satisfying

$$
\left\|f_{\varepsilon}(t)\right\|_{l} \leq k
$$

for $t \in\left[0, T_{0}\right]$. 
If in addition $f_{0}$ satisfies

$$
f_{\varepsilon}(0)=t_{\left(h_{\varepsilon}, h_{\varepsilon}\right)}+\varepsilon^{2} k_{\varepsilon}, \quad\left\|\partial_{x} h_{\varepsilon}\right\|_{2} \leq C \varepsilon, \quad \lim _{\varepsilon \rightarrow 0}\left\|h_{\varepsilon}-h\right\|_{2}=0,
$$

we get

Theorem 5. As $\varepsilon \rightarrow 0, f_{\varepsilon} \rightarrow f=\rho \cdot e$ weakly in $L^{\infty}\left(\left[0, T_{0}\right], H^{1}\right)$ and strongly in $C\left([0, T], L^{2}(x)\right)$ and the limit satisfies the heat equation:

$$
\partial_{t} \rho=\frac{1}{4} \partial_{x}^{2} \rho, \quad \rho(0, x)=\langle h, e\rangle .
$$

\section{Example, II (Broadwell model)}

We shall first describe the six-velocity model gas considered by Broadwell [17] and in [29] and then specialize it to one dimension. We consider the following equation:

$$
\varepsilon \frac{d F_{i}}{d t}+u_{i} \cdot \nabla F_{i}=\frac{B}{3 \varepsilon}\left(F_{i+1} F_{i+4}+F_{i+2} F_{i+5}-2 F_{i} F_{i+3}\right), \quad i=0, \ldots, 5
$$

Here $F_{i}$ are the densities of the particles with velocity

$$
u_{i}=\left(c \cos \left(\theta+i \frac{\pi}{3}\right), c \sin \left(\theta+i \frac{\pi}{3}\right)\right),
$$

and $B$ denotes the collision frequency.

We specialize (5.1) to solutions that do not depend on $y$, and $\theta=0$, and such that $F_{5}=F_{1}$ and $F_{4}=F_{2}$. We can write $(5.1)$ for $F_{1}, F_{2}, F_{3}$ and $F_{4}$ :

$$
\begin{aligned}
& \varepsilon \frac{d F_{0}}{d t}+\frac{d F_{0}}{d x}=\frac{2 B}{3 \varepsilon}\left(F_{1} F_{2}-F_{0} F_{3}\right), \\
& \varepsilon \frac{F_{1}}{d t}+\frac{1}{2} \frac{d F_{1}}{d x}=\frac{B}{3 \varepsilon}\left(F_{0} F_{3}-F_{1} F_{2}\right), \\
& \varepsilon \frac{d F_{2}}{d t}-\frac{1}{2} \frac{d F_{2}}{d x}=\frac{B}{3 \varepsilon}\left(F_{0} F_{3}-F_{1} F_{2}\right), \\
& \varepsilon \frac{d F_{3}}{d t}-\frac{d F_{3}}{d x}=\frac{2 B}{3 \varepsilon}\left(F_{1} F_{2}-F_{0} F_{3}\right) .
\end{aligned}
$$

By the form of second member we have $\left(\alpha_{0}, \alpha_{1}, \alpha_{2}, \alpha_{3}\right)=\left(\frac{1}{2}, 1,1, \frac{1}{2}\right)$ and the relation $(1,3)$ is satisfied. To get the equation (1.11)-(1.12) for the Broadwell's model we need some preparations. The space $\mathbb{M}$ of summational invariants consists of vectors $\phi=t_{\left(\phi_{1}, \phi_{2}, \phi_{3}, \phi_{4}\right)}$ satisfying $2 \phi_{1}+2 \phi_{4}=\phi_{2}+\phi_{3}$. Therefore $\mathbb{M}$ is spanned by $\left\{\phi_{1}, \phi_{2}, \phi_{3}\right\}$, where

$$
\phi_{1}=t_{(1,0,0,-1)}, \quad \phi_{2}=t_{\left(0,1,0 \frac{1}{2}\right)}, \quad \phi_{3}=t_{\left(0,0,1, \frac{1}{2}\right)}
$$

On the other hand a locally Maxwellian state is a vector $F=t_{\left(F_{0}, F_{1}, F_{2}, F_{3}\right)}>0$ satisfying $F_{2} F_{1}=F_{0} F_{3}$. Let $M=t_{(1,1,1,1)}$ be an absolute Maxwellian state. Set $F(t, x)=M+$ $\Lambda^{\frac{1}{2}} f(t, x)$ for $\Lambda=\operatorname{diag}(2,1,1,2)$ and substitute it into $(5.2)$ :

$$
\begin{aligned}
& f_{t}+\frac{V f_{x}}{\varepsilon}+\frac{L f}{\varepsilon^{2}}=\frac{1}{\varepsilon} \Gamma(f, f), \\
& f(t=0, x)=f_{0}(x),
\end{aligned}
$$


where $V=\operatorname{diag}\left(1, \frac{1}{2},-\frac{1}{2},-1\right)$ and

$$
\begin{aligned}
& L=-\frac{B}{3}\left(\begin{array}{cccc}
-2 & \sqrt{2} & \sqrt{2} & -2 \\
\sqrt{2} & -1 & -1 & \sqrt{2} \\
\sqrt{2} & -1 & -1 & \sqrt{2} \\
-2 & \sqrt{2} & \sqrt{2} & -2
\end{array}\right), \\
& \Gamma(f, f)=\frac{B}{3}\left(f_{2} f_{1}-2 f_{0} f_{3}\right) t_{(\sqrt{2},-1,-1, \sqrt{2})} .
\end{aligned}
$$

Since $N(L)=\Lambda^{\frac{1}{2}} \mathbb{M}$, a simple calculations gives the orthonormal basis $\left\{e_{i}, i=1,2,3\right\}$ for $N(L)$ :

$$
e_{1}=t_{\left(\frac{\sqrt{2}}{2}, 0,0,-\frac{\sqrt{2}}{2}\right)}, \quad e_{2}=t_{\left(\frac{1}{\sqrt{10}}, \frac{2}{\sqrt{5}}, 0, \frac{1}{\sqrt{10}}\right)}, \quad e_{3}=t_{\left(\frac{1}{\sqrt{15}},-\frac{1}{\sqrt{30}}, \frac{\sqrt{5}}{\sqrt{6}}, \frac{1}{\sqrt{15}}\right)} .
$$

By letting $\varepsilon \rightarrow 0$ in (5.3) and using the results of Section 1 , we get $f_{\varepsilon} \rightarrow f$ in $D_{t, x}^{\prime}$ (distribution sense) with $f=\rho \cdot e$, where

$$
\rho=t_{\left(\rho_{1}, \rho_{2}, \rho_{3}\right)}, \quad e=t_{\left(e_{1}, e_{2}, e_{3}\right)},
$$

and from (1.9) we get

$$
\rho_{3}=\frac{2 \sqrt{3}}{\sqrt{10}} \rho_{1}, \quad \rho_{2}=-\frac{2}{\sqrt{5}} \rho_{1} .
$$

Lemma 7. Let $f=\rho \cdot$ e satisfying (5.6), then

$$
\begin{aligned}
& \Gamma(f, f)=0, \\
& \langle V f, h\rangle=0 \quad \text { for any } \quad h \in N(L) .
\end{aligned}
$$

Proof. By using (5.5) and (5.6), we get (5.7). (5.8) is obtained by a direct calculations. This completes the proof.

Lemma 8. Let $f=\rho \cdot$ e satisfying (5.6), then

$$
\begin{aligned}
\left\langle L^{-1}\left(P^{\perp}\left(V e_{1}\right)\right), V f\right\rangle & =\frac{\rho_{1}}{2 B}, \\
\left\langle L^{-1}\left(P^{\perp}\left(V e_{2}\right)\right), V f\right\rangle & =-\frac{\rho_{1}}{4 B \sqrt{5}}, \\
\left\langle L^{-1}\left(P^{\perp}\left(V e_{3}\right)\right), V f\right\rangle & =\frac{\sqrt{6} \rho_{1}}{8 B \sqrt{5}} .
\end{aligned}
$$

Proof. The proof of this lemma follows from Lemma 7.

Using the results of Section 1 combined with Lemma 8, one gets:

Theorem 6. Let $f_{0} \in H^{1}(\mathbb{R})$, then there exists a positive constants $T_{0}$ and $k$ (depending only on $\left\|f_{0}\right\|_{l}$ ) such that the initial value problem (5.3) has a unique solution $f_{\varepsilon} \in L^{\infty}\left(\left[0, T_{0}\right], H^{1}(\mathbb{R})\right) \cap C\left([0, T], L^{2}(\mathbb{R})\right)$ satisfying

$$
\left\|f_{\varepsilon}(t)\right\|_{l} \leq k
$$

for $t \in\left[0, T_{0}\right]$. 
If in addition the initial condition satisfies:

$$
\begin{aligned}
& f_{\varepsilon}(0)=h_{\varepsilon}+\varepsilon^{2} k_{\varepsilon}, \quad L h_{\varepsilon}=0, \quad\left\|\Gamma\left(h_{\varepsilon}, h_{\varepsilon}\right)\right\|_{2} \leq C \varepsilon, \\
& \left\|\partial_{x} h_{\varepsilon}\right\|_{2} \leq C \varepsilon, \quad \lim _{\varepsilon \rightarrow 0}\left\|h_{\varepsilon}-h\right\|_{2}=0,
\end{aligned}
$$

one gets:

Theorem 7. As $\varepsilon \rightarrow 0, f_{\varepsilon} \rightarrow f=\rho \cdot e$ weakly in $L^{\infty}\left(\left[0, T_{0}\right], H^{1}\right)$ and strongly in $C\left([0, T], L^{2}(x)\right)$ and the limit satisfies the heat equation:

$$
\begin{aligned}
& \partial_{t} \rho_{1}=\frac{1}{4 B} \partial_{x}^{2} \rho_{1}, \quad \rho_{3}=\frac{2 \sqrt{3}}{\sqrt{10}} \rho_{1}, \quad \rho_{2}=-\frac{2}{\sqrt{5}} \rho_{1}, \\
& \rho_{1}(t=0)=\frac{\rho_{10}-\frac{2}{\sqrt{5}} \rho_{20}+2 \frac{\sqrt{3}}{\sqrt{10}} \rho_{30}}{3},
\end{aligned}
$$

where

$$
\rho_{i 0}=\left\langle h, e_{i}\right\rangle, \quad i=1,2,3 .
$$

Remark 5. One can remove the assumption $\left\|\Gamma\left(h_{\varepsilon}, h_{\varepsilon}\right)\right\|_{2} \leq C \varepsilon$ in (5.13). A price to be paid for this improvement is that: we see the solution of (5.2) in the form $F_{\varepsilon}=M+\varepsilon \phi(\varepsilon) f_{\varepsilon}$ with $\phi(\varepsilon)=O(\varepsilon)($ see $[12])$.

\section{References}

[1] Babovsky M, A Kinetic Multiscale Model, Math. Models Meth. Appl. Sci. 12 (2002), 209-234.

[2] Bardos C, Golse F and Levermore D, Sur les limites asymptotiques de la théorie cinétique conduisant a la dynamique des fluides incompressibles, C. R. Acad. Sci. Paris 309 (1989), $727-732$.

[3] Bardos C, Golse F and Levermore D, Fluid Dynamic Limits of Kinetic Equations I, Formal Derivations, Transp. Theory Statist. Phys. 21 (1992), 531-555.

[4] Bardos C, Golse F and Levermore D, Fluid Dynamical Limits of Discrete Velocity Kinetic Equations, in Advances in Kinetic Theory and Continuum Mechanics, Editor: Gatignol et Soubbaramayer R., Springer-Verlag, Berlin, New York, 1991, 57-71.

[5] Bardos C, Golse F and Levermore D, Fluid Dynamic Limits of Kinetic Equations: Convergence Proofs for the Boltzmann Equation, Comm. Pure Appl. Math. 46 (1993), 667-753.

[6] Bardos C and Ukai S, The Classical Incompressible Navier-Stokes Limit of the Boltzmann Equations, Math. Models Meth. Appl. Sci. 2 (1991), 235-257.

[7] Bellomo N and Kawashima S, The Discrete Boltzmann Equation with Multiple Collisions, Global Existence and Stability for the Initial Value Problem, J. Math. Phys. 31 (1990), 245253.

[8] Bellomo N and Gustafsson T, The Discrete Boltzmann Equation: A Review of the Mathematical Aspects of The Initial and Initial-Boundary Value Problems, Reviews in Math. Phys. 3 (1991), 137-162. 
[9] Bellouquid A, Limite asymptotique pour le Modèle de Carleman, C. R. Acad. Sci. Paris 321 (1995), 655-658.

[10] Bellouquid A, The Hydrodynamical Limit of the Nonlinear Boltzmann Equation, J. Transp. Theor. Stati. Phys. 28 (1999), 25-57.

[11] Bellouquid A, The Hydrodynamical Limit of Discrete Kinetic Equations, C. R. Acad. Sci. Paris 330 (2000), 951-956.

[12] Bellouquid A, Diffusive Limit for Nonlinear Discrete Velocity Models. To appear in Math. Models Meth. Appl. Sci., 13, (2003).

[13] Bellouquid A, Thèse de Doctorat, Université paris 7, 1996.

[14] Bonilla L and Soler J S, High-Field Limit of the Vlasov Poisson Fokker Plank System-A Comparison of Different Perturbation Methods, Math. Models Meth. Appl. Sci. 11 (2001), $1457-1468$.

[15] Bony J M, Existence globale à données de Cauchy Petites pour les modèles Discrets de l'Equation de Boltzmann, Preprint centre de Mathématiques Ecole Polytechnique 91128 palaiseau.

[16] Boltzmann L, Weitere Studien uber das Warmegleichgewicht unter Gas-molekulen, Sitzungs. Akad. Wiss. Wein 66 (1872), 275-370; in English: Further Studies on the Thermal Equilibrium of Gas Molecules, in Kinetic Theory, Vol. 2, Editor: Brush S G, Pergamon Press, London, 1966, 88-174.

[17] Broadwell J E, Shock Structure in a Simple Discrete Velocity Gas, The Physics of Fluids 7 (1964), 1243-1247.

[18] Cabannes H, The Discrete Boltzmann Equation (Theory and Applications), Lecture Notes, Univ. of California, Berkely, 1980.

[19] Caflish R and Papanicolaou G, The Fluid-Dynamical Limit of a Nonlinear Model Boltzmann Equation, Comm. Pure Appl. Math. 32 (1979), 589-616.

[20] Carlemann T, Problémes mathématiques dans la théorie cinétique des gaz. Appsala. Publications scientifiques de l'institut Mittag-Leffler, 1957.

[21] De Masi A, Esposito R and Lebowitz J L, Incompressible Navier-Stokes and Euler Limits of the Boltzmann Equation, Comm. Pure Appl. Maths. 42 (1989), 321-366.

[22] Diperna R J and Lions P L, On the Cauchy Problem for the Boltzmann Equation: Global Existence and Weak Stability Results, Ann. Math. 130 (1989), 321-366.

[23] Gatignol R, Théorie cinétique des gaz à répartition discrète de vitesses, Lecture Notes in Phys., Vol. 36, Springer-Verlag, New York, 1975.

[24] Golse F, Lions P, Perthame B and Sentis R, Regularity of the Moments of of the Solution of a Transport Equation, J. of Funct. Anal. 76 (1988), 110-125.

[25] Golse F and Levermore D, Stokes-Fourier and Acoustic Limits for the Boltzmann Equation: Convergence Proofs, Preprint, Ecole normale supérieure, DMA-01-12, 2001.

[26] Golse F and Saint-Raymond L, The Navier-Stokes Limit for the Boltzmann Equation, C. R. Acad. Sci. Paris 333 (2001), 897-902. 
[27] Gorsch D, Generalized Discrete Velocity Models, Math. Models Meth. Appl. Sci. 12 (2002), $49-75$.

[28] Hammouch J, These de l'Universite Paris 7, 1996.

[29] Harris S, Approach to Equilibrium in a Moderately Dense Discrete Velocity Gas, The Physics of Fluids 9 (1966), 1328-1334.

[30] Inoue K and Nishida T, On the Broadwell Model of the Boltzmann Equation for a Simple Discrete Velocity Gas, Appl. Math. Optim. 3 (1976), 259-272.

[31] Kawashima S, Global Existence and Stability of Solutions for Discrete Velocity Models of the Boltzmann Equation, Lecture Notes in Num. Appl. Anal., 1983, 59-85. Recent Topics in Nolinear PDE, Hiroshima, 1983.

[32] Kawashima S, Large-Time Behavior of Solutions of the Discrete Boltzmann Equation, Commun. Math. Phys. 109 (1987), 563-589.

[33] Kurtz T G, Convergence of Sequences of Semigroups of Nonlinear Operators with an Application to Gas Kinetics, Trans. Amer. Math. Soc. 186 (1973), 259-272.

[34] Lachowicz M, Asymptotic Analysis of Nonlinear Kinetic Equations The Hydrodynamic Limit, in Lecture Notes on the Mathematical Theory of the Boltzmann Equation, Editor: Bellomo N, World Sci., London, Singapore, 1995.

[35] Leray J, Sur le mouvement d'un liquide visqueux emplissant l'espace, Acta Math. 63 (1934), $193-248$.

[36] Lions P and Toscani G, Diffusive Limits for Finite Velocity Boltzmann Kinetic Models, Revista Mat. Iberoamer. 13 (1997), 473-513.

[37] Lions P and Masmoudi N, From Boltzmann Equation to the Equation of Incompressible Fluid Mechanics I, Arch. Rat. Mech. Anal. 158 (2001), 173-193.

[38] Maxwell J C, On the Dynamical Theory of Gases, Phil. Trans. Roy. Soc. London 157 (1866), 49-88; also in The Scientific Papers of James Clerk Maxwell, Vol. 2, Dover, New York, 1965, $26-78$.

[39] Tartar L, Existence globale pour un systeme semi lineaire de la theorie cinetique des gas, seminaire Goulaouic Schwartz, Ecole Polytechnique Palaiseau, France, 1975, 475-485. 